\title{
College PE Teaching Quality Evaluation Model Based on Multilevel Fuzzy Analysis Comprehensive Evaluation Method
}

\author{
Xiuhua $\mathrm{Wu}^{1}$, Xingyu Huang ${ }^{2}$ \\ 1.Department of Physical Education, School of Science and Technology, Gannan Normal \\ University, Ganzhou, China \\ w_u_xiuhua@126.com \\ 2.School of Physical Education, Gannan Normal University, Ganzhou, China
}

Keywords:multilevel fuzzy analysis; PE teaching quality; fuzzy judgment matrix

\begin{abstract}
In this article, we provide the quantitative evaluation to college PE teaching quality by using the multi-level fuzzy comprehensive evaluation method, and then the evaluation grades are presented. Combined the training target of the students, guiding ideology of the teachers and the practical teaching situation, we make out a set of evaluation index system. Then we get the fuzzy judgment matrix of factors reflecting PE teaching quality according after hierarchical classification and significance quantization. By questionnaire, the fuzzy judgment matrix is finally obtained. The digital fuzzy relation matrix will be calculated. Through multi-layer compound operation, the evaluated object belonging grade will be determined. This model can evaluate PE teaching quality more completely and scientifically.
\end{abstract}

\section{Introduction}

At present, many students have little time to do exercises and weak physical ability. They have poor physical quality and the present evaluation system can provide scientific and reasonable evaluation to the students, which lead to the weary emotion to PE lessons. So realizing the shortage and limitation is the necessity of the evaluation system reform of PE lesson. We should strengthen the development oriented function of the evaluation system. We should think highly of the individualism and the hardship of the students. The progress should be confirmed; the interest and enthusiasm of the students should be stimulated; the student physical quality overall evaluation system should be improved and the student comprehensive evaluation should be implemented and student self evaluation, mutual evaluation and teacher evaluation method needs completion. In this way, PE teaching can really fit for the college student situation.

The teaching quality evaluation is an effective measure to improve teaching quality, adjust teaching behavior, optimize teacher structure, improve the teaching level and make the faculty systematic and scientific. In recent years, our university promotes the transfer of the education, deepens the policy reform of staff and raises more requests to the teaching quality. By establishing the hierarchy diagram of PE teaching quality evaluation system, we construct fuzzy consistency judgment matrix and calculate the weights of the indexes. By analysis of the questionnaires of students, teachers and experts, we have obtained the fuzzy judgment matrixes and calculate the evaluation scores of different groups. Through the weighted calculation of the scores, we get the final results of the lessons.

\section{The Model Hypothesis and Symbol Definition}

\section{A. The Model Hypothesis}

In the evaluation of PE teaching quality model, the teachers can be honest in self evaluation and evaluate the advantage and disadvantage objectively; but they don't consider and care about others in mutual evaluation.

The student evaluation is implemented before the examination. The experts organize students to fill in the evaluation form carefully. And the students can present the evaluation practically and realistically according to their own will. 
All the questionnaires can be collected without alteration.

The expert evaluation is implemented by group experts. According to the observed lessons, student forum, homework, examination paper, teaching plan and teaching reports, the experts present the evaluation.

When the following situation appears, the teachers will be defined unqualified.

A. When a lot of teaching accidents happen;

B. If half of the students participating in the evaluation think the teaching effect unqualified, we can directly regard the teachers unqualified.

B. The Establishment of Symbol System and Relative formulas

According to the hierarchical model, we define the symbol system and relative formulas about the algorithm.

In the first grade evaluation indexes, suppose teacher self evaluation, teacher mutual evaluation, expert evaluation, and student evaluation as $A_{1}, A_{2}, A_{3}, A_{4}$. And we get the set of first grade index factors $A=\left\{A_{1}, A_{2}, A_{3}, A_{4}\right\}$.

In the second grade evaluation indexes, suppose teaching target, teaching content, teaching method, teaching quality, teaching effect and answering questions as $B_{1}, B_{2}, B_{3}, B_{4}, B_{5}, B_{6}$. Then we get the set of second grade index factors $B=\left\{B_{1}, B_{2}, B_{3}, B_{4}, B_{5}, B_{6}\right\}$.

In the third grade evaluation indexes, suppose the third grade indexes attached to second grade indexes $B_{i}(i=1,2,3,4,5,6)$ are $C_{i 1}, C_{i 2}, C_{i 3}, C_{i 4}, C_{i 5}, B_{i 6}$ which can be marked as $C_{i}\left\{C_{i 1}, C_{i 2}, C_{i 3}, C_{i 4}, C_{i 5}, B_{i 6}\right\}$. Apparently, if the elements in third grade indexes set $C_{i}$ attached to second grade indexes $B_{i}(i=1,2,3,4,5,6)$ can not reach 6 items, we will deal with them as follows: we will directly omit the shorted items in set $C_{i}$. For example, $C_{1}$ will be marked as $C_{11}, C_{12}$ which represents \{accuracy of teaching, truthfulness\}.

We decide there are four grades in our evaluation which are excellent (90-100), good (80-90), pass (60-80) and failed (0-60). They are marked as $V_{1}, V_{2}, V_{3}, V_{4}$. We also will establish evaluation set $V\left\{V_{1}, V_{2}, V_{3}, V_{4}\right\}$.

Determine the weights of the evaluation index system. In our model, weight is very important which can directly affect the result of the comprehensive evaluation. And our weight solution is a comparative matrix. Suppose there is a set $A=\left\{A_{1}, A_{2}, A_{3}, A_{4}, A_{5}, A_{6}\right\}$ and this set is different from first grade index factor set. We can establish a fuzzy consistency judgment matrix.

$$
A=\left(\begin{array}{llllll}
a_{11} & a_{12} & a_{13} & a_{14} & a_{15} & a_{16} \\
a_{21} & a_{22} & a_{23} & a_{24} & a_{25} & a_{26} \\
a_{31} & a_{32} & a_{33} & a_{34} & a_{35} & a_{36} \\
a_{41} & a_{42} & a_{43} & a_{44} & a_{45} & a_{46} \\
a_{51} & a_{52} & a_{53} & a_{54} & a_{55} & a_{56} \\
a_{61} & a_{62} & a_{63} & a_{64} & a_{65} & a_{66}
\end{array}\right)
$$

And $a_{i j}$ represents that factor $A_{i}$ and factor $A_{j}$ have fuzzy relation and the membership degree is that $A_{i}$ is more important than $A_{j}$.

\begin{tabular}{|l|l|}
\hline \multicolumn{1}{|c|}{ Criterion $a_{i j}$} & \multicolumn{1}{c|}{ Meaning } \\
\hline 1 & $A_{i}$ and $A_{j}$ have same impact. \\
\hline 3 & $A_{i}$ has little more impacts than $A_{j}$ \\
\hline 5 & $A_{i}$ has more impacts than $A_{j}$ \\
\hline
\end{tabular}




\begin{tabular}{|l|l|}
\hline 7 & $A_{i}$ has obvious more impacts than $A_{j}$ \\
\hline 9 & $A_{i}$ has definite more impact than $A_{j}$ \\
\hline $2,4,6,8$ & The impact proportion of $A_{i}$ and $A_{j}$ is between the neighboring two grades \\
\hline $1,1 / 2, \cdots, 1 / 9$ & The impact proportion of $A_{i}$ and $A_{j}$ is the converse number of the above number. \\
\hline
\end{tabular}

According to the relative theory, we can obtain the eigenvector $W$ corresponding to the maximum latent root and the weight vector of the set. In this article we use Matlab to resolve. At the same time we quote the definition of inconsistency and method of solve inconsistent matrix in Saaty's AHP. Saaty regards

$C_{i}=(d-n) /(n-1)$

as the consistency indexes. And $d$ is the maximum latent root of $A$ and $n$ represents the exponent number of $A$. We also introduce random consistency index $R_{i}$. The value is as below,

TABLE I. THE VALUE OF RANDOM CONSISTENCY INDEX $R_{i}$

\begin{tabular}{|l|l|l|l|l|l|l|l|l|l|l|l|}
\hline $\mathbf{n}$ & 1 & 2 & 3 & 4 & 5 & 6 & 7 & 8 & 9 & 10 & 11 \\
\hline$R_{i}$ & 0 & 0 & 0.58 & 0.90 & 1.12 & 1.24 & 1.32 & 1.41 & 1.45 & 1.49 & 1.51 \\
\hline
\end{tabular}

The proportion between consistency index CI and random consistency index $R_{i}$ of the same order is called consistency ratio $C R$ in the pair comparison matrix $(n>=3) A$. When

$\mathrm{CR}=\mathrm{CI} / \mathrm{RI}<0.1$,

we believe that the inconsistency of $A$ is within the permissible range; we can regard the eigenvector as the weight vector. According to the above illustration, we can get weight vector of first grade factor index vector set $W_{A}=\left[W_{A 1}, W_{A 2}, W_{A 3}, W_{A 4}\right]$.

The weight vector of second grade factor index set is $W_{B}=\left[W_{B 1}, W_{B 2}, W_{B 3}, W_{B 4}, W_{B 5}, W_{B 6}\right]$. Then we can obtain the weight vector of third grade factor index set

$$
\begin{array}{ccc}
W_{B 1}=\left[W_{11}, W_{12}\right] & W_{B 2}=\left[W_{21}, W_{22}, W_{23}, W_{24}\right] & W_{B 3}=\left[W_{31}, W_{32}, W_{33}, W_{34}, W_{35}\right] \\
W_{B 4}=\left[W_{41}, W_{42}, W_{43}, W_{44}\right] & W_{B 5}=\left[W_{51}, W_{52}, W_{53}, W_{54}, W_{55}\right] & W_{B 6}=\left[W_{61}, W_{62}\right]
\end{array}
$$

We define the comprehensive judgment matrix of fourth level. $R_{j k}$ ( $j=1,2,3,4$ respectively represent the comprehensive judgment matrixes of teacher self evaluation, teacher mutual evaluation, expert evaluation and student evaluation questionnaires). According to the original information from the questionnaire, we can get the excellent ratio, good ratio, pass ratio and failed ratio in 6 third index set of the class evaluation by Excel, which are shown in $R_{k}(k=1,2,3,4,5,6)$.

$$
R_{k}=\left(\begin{array}{llll}
r_{k 11} & r_{k 12} & r_{k 13} & r_{k 14} \\
r_{k 21} & r_{k 22} & r_{k 23} & r_{k 24} \\
r_{k 31} & r_{k 32} & r_{k 33} & r_{k 34} \\
r_{k 41} & r_{k 42} & r_{k 43} & r_{k 44}
\end{array}\right)
$$

$r_{k 11}, r_{k 12}, r_{k 13}, r_{k 14}$ represent the excellent ratio, good ratio, pass ratio and failed ratio of third factor $C_{k 1}$ attached to the second index factor.

7) We define the comprehensive judgment matrix $P$ in the third level. The comprehensive judgment matrix of each factor in the third level is $P_{j i}=R_{j i} * W_{b i}(i=1,2,3,4,5,6)$.

Finally we can obtain $P_{j}=\left[p_{j 1}, p_{j 2}, p_{j 3}, p_{j 4}, p_{j 5}, p_{j 6}\right]^{T} .(j=1,2,3,4$ represent the fourth level judgment matrixes of teacher self evaluation, teacher mutual evaluation, expert evaluation and student evaluation. And then we can get the comprehensive judgment matrix in the third level.)

The comprehensive judgment matrixes in the second level are $S_{1}, S_{2}, S_{3}, S_{4}$. We can calculate the comprehensive judgment matrixes $S_{1}, S_{2}, S_{3}, S_{4}$ for teacher self evaluation, teacher mutual evaluation, expert evaluation and student evaluation in the second level. And the calculation method is 


$$
\begin{gathered}
S_{j}=W_{b} * P_{j}=\left[w_{1}, w_{2}, w_{3}, w_{4}, w_{5}, w_{6}\right] *\left[p_{j 1}, p_{j 2}, p_{j 3}, p_{j 4}, p_{j 5}, p_{j 6}\right]^{T} \\
\text { Table II. } \quad \text { The table of evaluation grades and scores }
\end{gathered}
$$

\begin{tabular}{|l|l|l|l|l|}
\hline \multicolumn{1}{|c|}{ Grades } & Excellent & Good & Pass & Failed \\
\hline Score Section & $90-100$ & $80-90$ & $60-80$ & $45-60$ \\
\hline Representative Score & 95 & 85 & 70 & 53 \\
\hline
\end{tabular}

The vector of the score is $Q=[95,85,70,53]^{\mathrm{T}}$.

Calculate the scores $Q_{1}, Q_{2}, Q_{3}, Q_{4} Q_{i}=S_{i}{ }^{*} Q \quad(i=1,2,3,4)$ of teacher self evaluation, teacher mutual evaluation, expert evaluation and student evaluation in the second level.

The final Re sult $=\left[Q_{1}, Q_{2}, Q_{3}, Q_{4}\right] * W_{A}=\left[Q_{1}, Q_{2}, Q_{3}, Q_{4}\right] *\left[W_{A 1}, W_{A 2}, W_{A 3}, W_{A 4}\right]$

\section{The Algorithm Analysis and Resolution}

Firstly we handle the questionnaire of the students and get the value of student evaluation $S_{4}$.

Based on the student questionnaire, we implement the grade evaluation of the $22^{\text {nd }}$ item in the fourth level of PE teaching hierarchical model. Using EXCEL to count and analyze the result of the evaluation, we can obtain 5 fuzzy judgment matrixes attached to the $3^{\text {rd }}$ level.

The we can get the following teaching target fuzzy judgment matrix $R_{41}$ (4 represents the model is based on the data of the student questionnaire.) $R_{41}=\left(\begin{array}{llll}0.18 & 0.37 & 0.33 & 0.12 \\ 0.21 & 0.44 & 0.21 & 0.14\end{array}\right)$

Similarly, we can get teaching content fuzzy judgment matrix $R_{42}$, teaching method fuzzy judgment matrix $R_{43}$, teaching quality fuzzy judgment matrix $R_{44}$, teaching effect fuzzy judgment matrix $R_{45}$ and answering questions fuzzy judgment matrix $R_{46}$.

$$
\begin{aligned}
R_{42}= & \left(\begin{array}{llll}
0.30 & 0.55 & 0.15 & 0.00 \\
0.23 & 0.43 & 0.34 & 0.00 \\
0.19 & 0.40 & 0.33 & 0.08 \\
0.21 & 0.33 & 0.46 & 0.00
\end{array}\right) \quad R_{43}=\left(\begin{array}{llll}
0.23 & 0.4 & 0.32 & 0.01 \\
0.25 & 0.32 & 0.39 & 0.04 \\
0.44 & 0.34 & 0.22 & 0.00 \\
0.23 & 0.46 & 0.29 & 0.02 \\
0.20 & 0.41 & 0.28 & 0.11
\end{array}\right) \\
R_{46} & =\left(\begin{array}{llll}
0.80 & 0.20 & 0.00 & 0.00 \\
0.76 & 0.24 & 0.00 & 0.00
\end{array}\right)
\end{aligned}
$$

Determine the weight vectors of second grade indexes and third grade indexes. Taking two factors affiliated to the teaching target as an example, we should first build up pair comparison matrix.

$$
B_{1}=\left(\begin{array}{ll}
1 & 1 \\
1 & 1
\end{array}\right)
$$

According to the above formula, we can calculate the weight vector $W_{B 1}=[0.5,0.5]$. According to the character of PE teaching, we present the corresponding pair comparison matrix and resolve the corresponding weight vector as follows,

$$
\begin{aligned}
& W_{B 2}=[0.25,0.25,0.25,0.25] \quad W_{B 3}=[0.3,0.25,0.25,0.05,0.1] \\
& W_{B 4}=[0.2,0.3,0.3,0.2] \quad W_{B 5}=[0.2,0.2,0.1,0.2,0.2,0.1] \quad W_{B 6}=[0.4,0.6]
\end{aligned}
$$

All the pair vector matrixes should satisfy the consistency judgment standard which is $C R<0.1$.

As to the second index, the pair comparison matrix is judgment matrix which is shown below,

$\left(\begin{array}{llllll}1.0000 & 0.5000 & 0.3333 & 0.5000 & 0.2500 & 2.0000 \\ 2.0000 & 1.0000 & 2.0000 & 2.0000 & 3.0000 & 3.0000 \\ 3.0000 & 0.5000 & 1.0000 & 2.0000 & 0.3333 & 2.0000 \\ 2.0000 & 0.5000 & 0.5000 & 1.0000 & 0.3333 & 2.0000 \\ 4.0000 & 0.3333 & 3.0000 & 3.0000 & 1.0000 & 4.0000 \\ 0.5000 & 0.3333 & 0.5000 & 0.5000 & 0.2500 & 1.0000\end{array}\right)$

The calculation result is $C R=0.0783$ which satisfies consistency request. The weight vector is $W_{B}=(0.0837,0.3121,0.1551,0.1128,0.2728,0.0635)$ As to the first level index, the pair judgment

matrix of it is $\left(\begin{array}{llll}1.0000 & 2.0000 & 0.2500 & 0.2000 \\ 0.5000 & 1.0000 & 0.5000 & 0.3333 \\ 4.0000 & 2.0000 & 1.0000 & 0.5000 \\ 5.0000 & 3.0000 & 2.0000 & 1.0000\end{array}\right)$


After the calculation, we get $C R=0.0878, \quad W_{A}=(0.1215,0.1127,0.2928,0.4730)$

Calculate the comprehensive judgment matrix $P$ of second level index. According to the above formula $P_{j i}=R_{j i} * W_{b i}(i=1,2,3,4,5,6)$, we can get the following results one by one.

$$
\begin{array}{lllll}
P_{41}=\left[\begin{array}{llllll}
0.1950 & 0.4050 & 0.2700 & 0.1300
\end{array}\right] & P_{42}=\left[\begin{array}{lllll}
0.2325 & 0.4275 & 0.3200 & 0.0200
\end{array}\right] \\
P_{43}=\left[\begin{array}{llllll}
0.2830 & 0.3695 & 0.3050 & 0.0305
\end{array}\right] & P_{44}=\left[\begin{array}{lllll}
0.2960 & 0.3980 & 0.2660 & 0.0400
\end{array}\right] \\
P_{45}=\left[\begin{array}{llllll}
0.3205 & 0.4180 & 0.2565 & 0.0250
\end{array}\right] & P_{46}=\left[\begin{array}{lllll}
0.7760 & 0.2240 & 0 & 0
\end{array}\right]
\end{array}
$$

Finally, the comprehensive judgment matrix of second level indexes can be obtained.

$$
P_{4}=\left(\begin{array}{cccc}
0.195 & 0.405 & 0.270 & 0.130 \\
0.233 & 0.428 & 0.320 & 0.020 \\
0.283 & 0.370 & 0.305 & 0.305 \\
0.296 & 0.398 & 0.266 & 0.04 \\
0.321 & 0.418 & 0.257 & 0.025 \\
0.776 & 0.224 & 0 & 0
\end{array}\right)
$$

We can get student evaluation comprehensive judgment matrix $S_{4}=W_{B} * P_{4}$ in the first level factors. By using Matlab, we calculate $S_{4}=\left[\begin{array}{llll}0.3029 & 0.3978 & 0.2698 & 0.0332\end{array}\right]$

(5)Calculate the evaluation score $Q_{4}$ based on student questionnaire. $Q_{4}=S_{4} * Q$ means

$$
Q_{4}=\left[\begin{array}{llll}
0.3029 & 0.3978 & 0.2698 & 0.0332
\end{array}\right]^{*}[95 \neg 85 \neg 70 \neg 53]^{\mathrm{T}}=83.2341
$$

Similarly, we can calculate the evaluation scores $S_{1}, S_{2}, S_{3}$ of teacher self evaluation, teacher mutual evaluation and expert evaluation.

\section{Conclusion}

We establish the structure diagram of college PE teaching quality evaluation system by using AHP. According to the consistency test of comparison matrix, we can provide the weights of all the indexes, which can avoid the subjective decision. After systematically analyzing and standardized handling the information from the questionnaires, the final scores of PE teaching quality evaluation are presented. In a word, this model can scientifically and objectively evaluate the PE teaching quality, which can show the spirit of our college transition and the request of talent training plan (3 foundations and 4 capabilities).

\section{References}

[1] Sheng Jinbao, Feng Jingyu and Peng Xuehui, Risk Analysis Method of Small Reservoirs [J], Hydro Engineering Journal, 2008,3(1):28-35.

[2] Liu Yalian, Zhou Cuiying, Fuzzy Hierarchical Comprehensive Evaluation of Embankment Dam Safety and Its Application [J], Hydropower, 2010,36 (5):38-41.

[3] Yu Shaowei, The Establishment of Fuzzy Membership Function Based on Intervals [J], Shandong University Journal, 2010,(6):106-110.

[4] Wang Zhitao, Jiang Chao and Jiang Xiaolin, Research on Embankment Risk Comprehensive Evaluation Method Based on Fuzzy Theory [J], Water Conservation and Construction Engineering Journal, 2011,9(4):27-30.

[5] Ma Fuheng, Liu Chengdong and Xiang Yan, Dam Risk Comprehensive Evaluation Standards and Its Applicability [J], Hehai University Journal, 2008,36(5):610-614.

[6] Li Chunlei, Li Xiaolu, Multi Level Fuzzy Comprehensive Evaluation Research of Concrete Dam Safety [J], Hydropower, 2010,36(10):93-95.

[7] Chen Dongfeng, Lei Yingjie and Pan Hanjin, Radar Emitter Recognition Based on Fuzzy Weighted Method [J], Modern Defense Technology, 2008,33(6):57-59. 